\title{
Measurements of Neutrons In A Mixed Gamma- Neutron Field Using Three Different Types of Detectors
}

\section{B LEAL ${ }^{1}$, F. CASTILLO ${ }^{2 \dagger}$, J GUTIERREZ ${ }^{1}$, JI GOLZARRI ${ }^{3}$, I GAMBOA- DEBUEN $^{1}$, G. ESPINOSA ${ }^{3}$ H MARTÍNEZ ${ }^{2}$}

${ }^{1}$ Instituto de Ciencias Nucleares, Universidad Nacional Autónoma de México, Apartado Postal 70-543, 04510, Ciudad Universitaria, México D. F., México

${ }_{2}^{2}$ Laboratorio de Espectroscopia, Instituto de Ciencias Físicas, Universidad Nacional Autónoma de México, Apartado Postal 48-3, 62251, Cuernavaca Morelos, México

${ }^{3}$ Instituto de Física, Universidad Nacional Autónoma de México, Apartado Postal 20 364, 01000, Ciudad de México, México

\section{Email:}

Published online: August 07, 2017

The Author(s) 2017. This article is published with open access at www.chitkara.edu. in/publications

\begin{abstract}
A linear electron accelerator for medical use is a device for the treatment of tumors by collimated beams of electrons and/or photons. These accelerators are devices that employ electromagnetic waves of high frequency, to accelerate electrons that are used directly in the treatment of superficial tumors, or, if they are made to hit them on an appropriate target, they can produce photons of high energy destined to the treatment of deeptumors.Depending on the energy of the electrons and photons and the materials that make up the head of the accelerator and the target, this equipment will produce in addition to the aforementioned radiation, neutron fields of regular intensity. It is necessary to estimate the equivalent dose due to the neutrons themselves, the doses due to the gamma field of neutron capture, produced by the capture of thermal neutrons in the concrete of the bunker, and the gamma doses due to phenomena of neutron activation of elements of the own accelerator.

It is therefore important to be able to measure (detect, quantify, dose, etc.) both photons and neutrons in these cases and others more. In this work we use three different detectors, namely a scintillator-photomultiplier system, a fast reading dosimeter and bubble detector. The idea is to measure the radiation separately and compare their results.
\end{abstract}

Journal of Nuclear

Physics, Material Sciences, Radiation and Applications Vol-5, No-1, August 2017 pp. 223-235 
Leal, B

Castillo, F

Gutierrez, J

Golzarri, JI

Gamboa-deBuen, I

Espinosa, G

Martínez, $\mathrm{H}$

The results obtained were the mixed gamma-neutron field spectrum, the dose due only to neutrons obtained by the bubble detectors, which is compared to the dose obtained by the second fast reading dosimeters (model 884), plus the dose obtained by the first dosimeters (model 609) and finally the dose obtained by the Victoreen dosimeter.

Keywords: Detectors, Gammas, Neutrons, Dosimeters, Dose

\section{INTRODUCTION}

Neutrons are indirectly ionizing radiation that causes, at the same absorbed dose, biological effects more important than those produced by photons. Therefore, they are particles that must be taken into account in the dosimetry of personnel exposed to radiation. One of the facilities where the most important neutron fields are located is the interior of the containment buildings of nuclear power plants and nuclear fuel treatment units. Also found are neutron fields in cosmic radiation and in the vicinity of particle accelerators, such as those used for medical applications.

While it is true that in installations such as nuclear power plants, thecontribution of neutrons to the personal dose is in most cases negligible,there are cases where there is a significant risk of neutron irradiation, such as an intervention in the containment area during reactor operation.

Another field where it is important to have very well determined the neutron field is in medicine, for example, machines like the LINAC. On the one hand, the radioprotection of the personnel working in the installation requires that the radiation fields, including the neutrons, present are perfectly characterized. In addition, the recommendations of the International Commission on Radiological Protection, published in its publication ICRP103 [1], take into account for the first time the radioprotection of patients undergoing treatments with ionizing radiation, and in particular that of patients submitted to radiotherapy treatments in linear electron accelerators (LINACs). Among the new recommendations is that of limiting the doses due to the radiation that can receive those healthy organs next to the treated tumor.

The neutron photo-production reactions that occur in the heavy materials (iron, lead, tungsten, ...) that constitute the gantry and collimators of the accelerator itself, give rise to a neutron field that occupies the entire irradiation room and which consequently contributes to the radiation dose to the tissues and organs of the patient outside the treated tumor. Most radiotherapy treatments are currently performed with energy potentialsranging from 15 to $18 \mathrm{MV}$, so this neutron dose must be taken into account. In addition, the survival of patients undergoing radiotherapy has increased in recent years and 
it is not negligible that a treated patient will, in the long term, appear to have a new tumor in an organ other than that originally treated, which could be radio -induced.

Other particle accelerators for medical, industrial or research applications (cyclotrons for the production of radiopharmaceuticals used in nuclear medicine, synchrotrons used for the modification of structures of materials or for medical applications, high energy accelerators for researchcan also originate neutron fields by mechanisms similar to those of LINAC, so that in all this type of installations the dosimetric control of the neutron component of the radiation must be correctly carried out.

One of the problems associated with neutron dosimetry is that neutron fields are usually accompanied by other components of radiation, often much more intense than the neutron component although their contribution to the total dose may be of the same order of magnitude. The main problem with neutron dosimetry is that the neutron weighting factors, which allow the calculation of the equivalent dose from the absorbed dose, depend strongly on the energy of the neutrons, and therefore it is not advisable to carry out its dosimetry without knowing the energy spectrum of the neutron field to be detected, at least approximately. This leads to the fact that to perform the dosimetry correctly it is necessary to have spectrometric information. In addition, the detection of neutrons encounters the added problem that, in the case of neutral particles, they do not directly produce a measurable signal in the detectors, but the detection mechanisms are based on the secondary charged particles originated in the interaction of the same with the different components of the absorbents.

\section{EXPERIMENTAL SETUP}

In this work, we usethe electronical system mounted as in different laboratoriesto obtainthe neutron spectrum from the Am241/Be source[2, 3, and 4].The scintillator-photomultiplier system consists of a cylindrical Bicron(C) BC-400 scintillator coupled to Hamamatsu(C) R1250, No. RA2457 photomultiplier, powered by two C4840 and three HTV C762-01 Hamamatsu(C power supplies, through Ortec (C) 269 photomultiplier bases. The scintillator has a $120 \mathrm{~mm}$ diameter, and is $50 \mathrm{~mm}$ high, and he is coupled to the photomultiplier by a silicon interface. The BC-400 plastic scintillator is known to produce a light output in the range of $400-500 \mathrm{~nm}$, with a peak about $425 \mathrm{~nm}$. The R1250 Hamamatsu(C) photomultipliers, on the other hand, they are sensitive between $300 \mathrm{~nm}$ and $650 \mathrm{~nm}$, respectively, with peak sensitivity at $420 \mathrm{~nm}$, so they are an ideal match for the BC-400 series.
Measurements of Neutrons In A Mixed Gamma-

Neutron Field

Using Three

Different Types of

Detectors

$+$


Leal, B

Castillo, F

Gutierrez, J

Golzarri, JI

Gamboa-deBuen, I

Espinosa, G

Martínez, $\mathrm{H}$

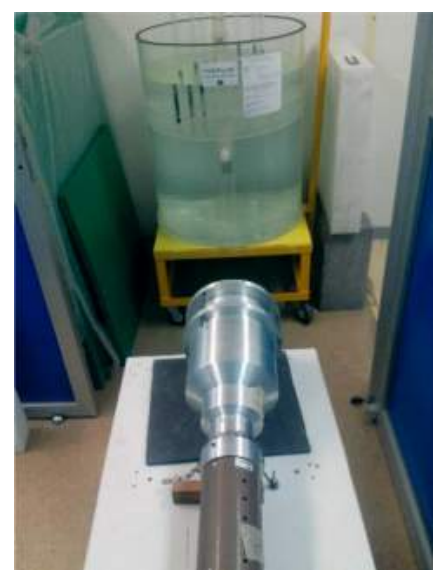

Figure 1: Geometrical arrangement of the BC-400 detector

The first step is to verify the scintillation spectrometer using an oscilloscope to check the type and form of the pulses. The system was mounted as can be seenin the Figure 1, where at the bottom the Visiflux contains the source in the middle of the water and in front it is shown the detector. For comparison two crystals are proposed, the BC-400 and the NE213 scintillator systems. The distance from source to detector were $100 \mathrm{~cm}$ collimating the beam and directed to the face of the detector, then between the source and the detectors will be used plates of lead and polyethylene to compare the signal. Due to the intensity of the source and the physics in the neutron behavior, the contribution of the neutron source in ambient background was present all the time in the laboratory.

The Bubble Detector Spectrometer (BDS) have six different energy thresholds ranging from $10 \mathrm{keV}$ to $10 \mathrm{MeV}$ [5, 6, 7, 8, and 9]. The number of bubbles obtained in each measurement is related to the dose (standardized response $\mathrm{R}$ ) equivalent neutrons through sensitivity $(\mathrm{b} / \mu \mathrm{Sv})$ and with the neutron flux (neutrons per unit area) through a relationship that is provided by the manufacturer. Bubble detectors were used with six different answers $(0.11$ b/ $\mu \mathrm{Sv}, 0.14 \mathrm{~b} / \mu \mathrm{Sv}, 0.17 \mathrm{~b} / \mu \mathrm{Sv}, 0.093 \mathrm{~b} / \mu \mathrm{Sv}, 0.051 \mathrm{~b} / \mu \mathrm{Sv}$ ) [6]. As can be seen in Figure 2, the detectors were placed inside the water container at $4 \mathrm{~cm}$ distance. We used several times to achieve low statistical error and to compare the response during the measurements. It was also measure the bare-naked beam from the source to analyze the contributions of thermal neutron. Following the equations 1 and 2, it is possible to construct the spectra thru neutron fluence.

$$
N_{i}=\frac{R_{i}-\sum_{j=i+1}^{6} \sigma_{i j} N_{j}}{\sigma_{i i}} \quad \operatorname{con} N_{7}=0
$$




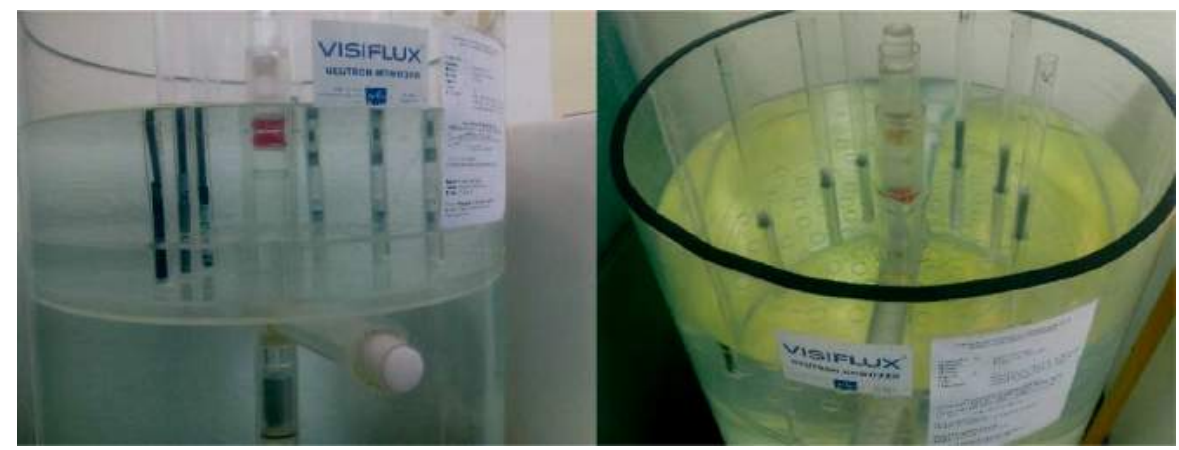

Measurements of Neutrons In A Mixed Gamma-

Neutron Field

Using Three

Different Types of

Detectors

Figure 2: Visiflux with the holder and two kind of detectors.

$$
R_{i}=\sum_{j=i}^{6} \sigma_{i j} N_{j}
$$

Where Ni is the neutron fluence for each detector, $\mathrm{R}_{\mathrm{i}}$ is the quotient of bubble's number and the sensitivity and $\sigma_{i j}$ is the neutron cross section.

The arrangement is the same for both BDS and DLD, using different times depending on whether they are thermal or gamma ray detectors, the objective isto obtain the linear response, the reproducibility and the sensibility of the detectors. The distance to be used are in the range from 2 to $14 \mathrm{~cm}$ and times from 8 to $240 \mathrm{~min}$. In every measure the charge was set to 0 to evaluate the response.

The direct reading dosimeter (DLD) is the size of a pocket pen, has a carbon fiber electroscope with an iron chamber for the detection and indication of integrated exposure to gamma radiation and neutrons. It has a thin wall that allows penetration and the detection of radiation. The dosimeters used are:Thermal neutron dosimeter, model 609, Equivalent dosimeter (gammas + rapid neutrons) model 884 and Gammas dosimeter model 5415 (Victoreen).

To obtain the sensitivity of the DLDs it is necessary to calculate the specific gamma constant for the radionuclides used in the experiment by equation 3

$$
\Gamma=19.38\left[\sum_{i=1}^{n} K_{i} E_{i}\left(\frac{1 / \varepsilon_{n}}{\dot{A}}\right)_{E_{i}}, \text { air }\right]\left[\frac{R m^{2}}{C i h}\right]
$$

Where $\Gamma$ is the specific constant, $K_{i}$ is the emission's probability, $E_{i}$ is the photon's energy, $\frac{\mu_{e m}}{\rho}$ is the mass energy-absorption coefficient. 
Leal, B

Castillo, F

Gutierrez, J

Golzarri, JI

Gamboa-deBuen, I

Espinosa, G

Martínez, $\mathrm{H}$

To obtain the calibration factor it is necessary to calculate the exposure rate at different distances by equation 4

$$
\dot{X}=\frac{\Gamma A}{d^{2}}
$$

where $\dot{X}$ the exposure rate is $\Gamma$ is the specific constant and $\mathrm{d}$ is the source to detector distance.

In Table 1 are shown the DLDs used in the experiment, where two of them are for thermal neutrons and three for gamma rays. Time of measure needs to guarantee that the fiber in the DLDs are in the middle of the range, so the uncertainty in all the dosimeters is the same.

For the last part, we used a single channel analyzer (Berthold Nuclear Spectrometer LB 2040) with a BF3 tube (Berthold LB6400) and a preamplifier (Charge sensitive LB 2008) to measure the spectra of thermal neutrons $[10,11]$. Figure 3 shows the BF3 detector and the polyethylene moderator. The measures were done at $1 \mathrm{~m}$, in three conditions bare-

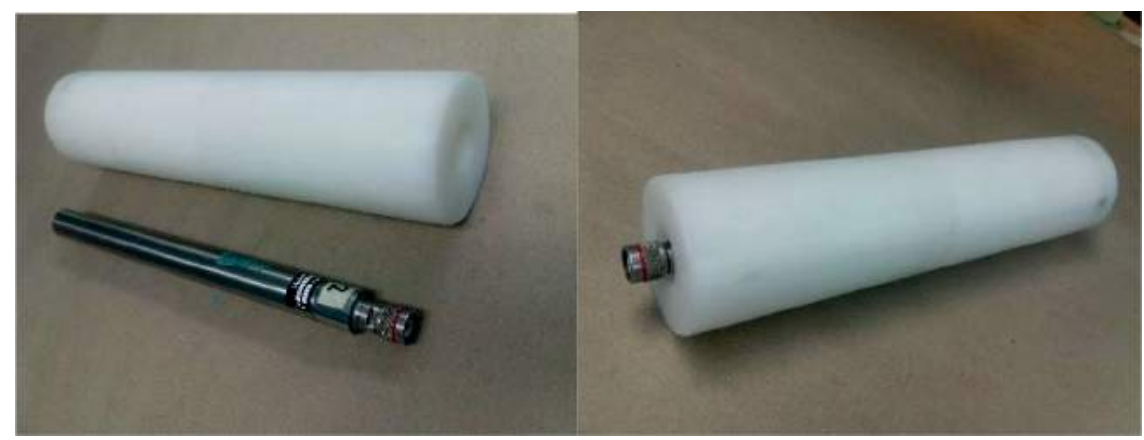

Figure 3: BF3 tube and moderator.

Table 1: Characteristics of the five DLD.

\begin{tabular}{llllll}
\hline Label & D1 & D2 & D3 & D4 & D5 \\
\hline $\begin{array}{l}\text { Serial } \\
\text { number }\end{array}$ & A040262 & 1515017 & 166843 & 110225 & A060618 \\
Range & $\begin{array}{l}0 \text { to } 200 \\
\mathrm{mR}\end{array}$ & 0 to $200 \mathrm{mR}$ & 0 to $200 \mathrm{mR}$ & 0 a $120 \mathrm{mrem}$ & 0 a $120 \mathrm{mrem}$ \\
Field & gamma & gamma & gamma & $\begin{array}{l}\text { Thermal } \\
\text { neutrons }\end{array}$ & $\begin{array}{l}\text { Thermal } \\
\text { neutrons }\end{array}$ \\
\hline
\end{tabular}


naked detector, detector with polyethylene moderator and a measure with the source in its shield.

\section{RESULTS}

From the modules used, no defective signals were observed on the oscilloscope and cables and connectors were checked to avoid false contacts. Figure 4 and 5 shows the spectra obtained with the two detectors, BC-400 and NE-213, in the case where the detectors were placed in front of the source in the collimated beam and without attenuators the spectra are similar, the first peak corresponds to the signal of the photons and from there the neutron spectrum is shown. To verify the behavior of the spectra two plates, one of lead and another of borated polyethylene, were placed between the source and the detector. The decrease due to neutron attenuation and moderation by the plates in the contribution of both gamma rays and neutrons is observed.

For the bubble detectors, we used 18 tubes and count the bubbles manually, for each set of thresholds the equations 1 and 2 were used.In Figure 6 we present the BDS before and after the irradiation with neutrons from the Am-241/Be source, the color in the background helped us to count the bubbles using a two-dimension photographic image, some tubes did not present bubbles at all.

The neutron spectra measured at 4 and $26 \mathrm{~cm}$ from the source are shown in figure 7. As expected the spectra as a function of water thickness changes not only in altitude but in shapebecause the neutron fluence change due to moderation and absorption inside the water. One parameter that cannot be controlled was the temperature in the laboratory, the manual specified that

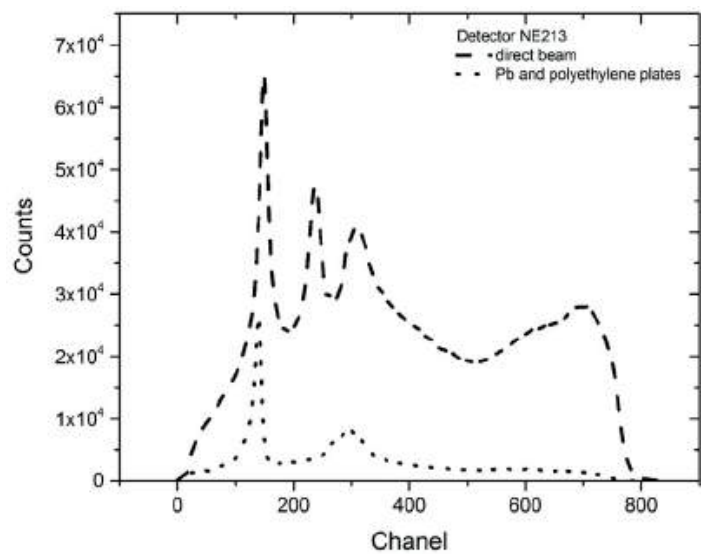

Figure 4: Neutron spectra measured with NE-213.
Measurements of Neutrons In A Mixed Gamma-

Neutron Field

Using Three

Different Types of

Detectors

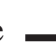


Leal, B

Castillo, F

Gutierrez, J

Golzarri, JI

Gamboa-deBuen, I

Espinosa, G

Martínez, $\mathrm{H}$

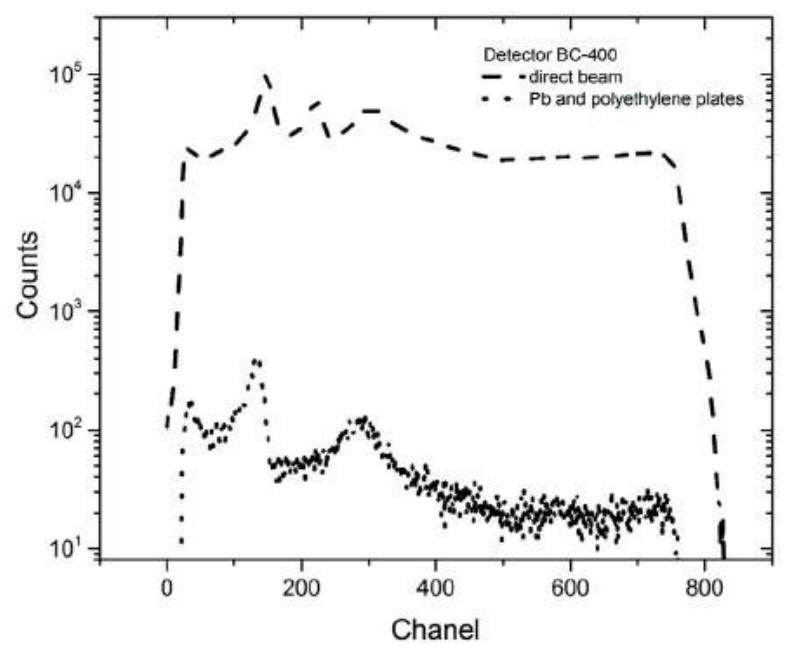

Figure 5: Neutron spectra measured with BC-400.

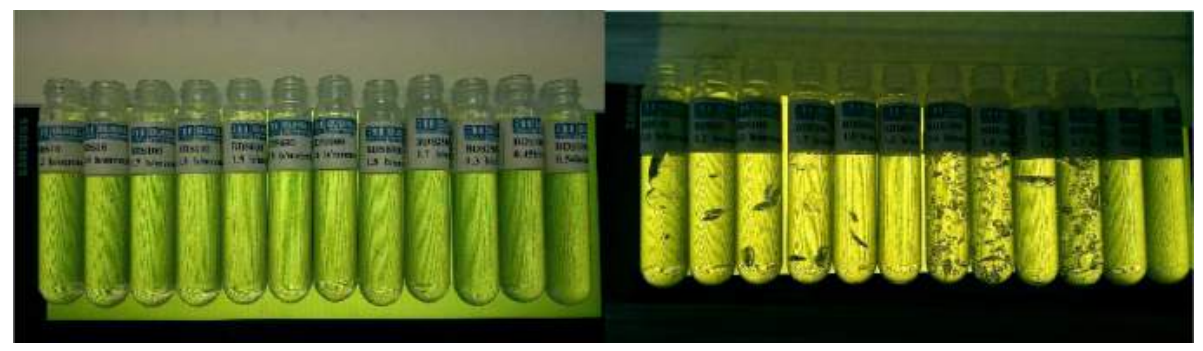

Figure 6: BDS before and after irradiation with neutrons

all the measures had to be done at $20^{\circ} \mathrm{C}$, the spectra at $26 \mathrm{~cm}$ was obtained at $25^{\circ} \mathrm{C}$. This could lead to a change in the response of bubbles detectors.

With the sensitivity of the BDS, we obtained the dose equivalent Hnear the source, then by dividing $\mathrm{H}$ per time we get the dose equivalent rate, which can be extrapolated to a different distance. Table 2 shows the data with which $\mathrm{H}$ was calculated, the number of bubbles is the average of three measurements performed under identical conditions, it should be noted that the measurements were made $4 \mathrm{~cm}$ apart from the source for a time of 45 minutes. According to current Mexican regulations [12], the limit of the dose equivalent for public is set at $5 \mathrm{mSv}$ per year.

If we add the contributions of each energy threshold and then sum the average dose equivalent of the complete spectrum, we obtained a value of $2.16 \mathrm{mSv} / \mathrm{h}$. Whereas for a distance of $26 \mathrm{~cm}$ that corresponds to the outer 




Figure 7: Spectra from the Am-241/Be source measured with bubbles detectors.

part of the shield, the dose equivalent rate of the complete spectrum is $61 \mu \mathrm{Sv} / \mathrm{h}$. Using a Bonner sphere detector we get a reading of $70 \mu \mathrm{Sv} / \mathrm{h}$ value similar of that obtained by the bubble detectors. Table 3 shows the values obtained for the distance of $26 \mathrm{~cm}$ and $17 \mathrm{~h}$ of irradiation.

The gamma exposure rates measured as a function of distance with DLDs are presented in figure 8 , as well as a theoretical calculation with eq. 4 , as can be seen, the exposure decreases rapidly as function of thickness. The exposure rate measured compared with the calculation has a maximum difference of about $9 \%$ for detector D1, detector D2 has a difference of $16 \%$ at $2 \mathrm{~cm}$ and around $9 \%$ for distances greater than $2 \mathrm{~cm}$ for detector D2 and between 15 and $28 \%$ for detector D3.Figure 9 shows the behavior of DLD4 and DLD5 for thermal neutrons, for comparison we fit an exponential decay equation, showing that no points match the attenuation.

In the case of thermal neutrons, the laboratory background was measured with the source inside its shield but without a polyethylene cover, then the collimated beam was left directly on the naked detector and finally a polyethylene cover was placed on the detector, all measurements were made one meter from the source. Figure 10 shows that both background and bare
Measurements of Neutrons In A Mixed Gamma-

Neutron Field

Using Three

Different Types of

Detectors 
Leal, B

Castillo, F

Gutierrez, J

Golzarri, JI

Gamboa-deBuen, I

Espinosa, G

Martínez, $\mathrm{H}$

Table 2: Dose equivalent at $4 \mathrm{~cm}$ from the source.

\begin{tabular}{ccccc}
\hline $\begin{array}{c}\text { Energy } \\
{[\mathrm{keV}]}\end{array}$ & Bubbles & $\begin{array}{c}\text { Sensitivity } \\
\text { bub/ } \mu \mathrm{Sv}\end{array}$ & $\begin{array}{c}H \\
{[\mu \mathrm{Sv}]}\end{array}$ & {$[\mu \mathrm{Sv}]$} \\
\hline 10 & 0 & 0.1 & 0 & 6.5 \\
10 & 1 & 0.11 & 9.1 & \\
10 & 2 & 0.19 & 10.5 & 26.8 \\
100 & 4 & 0.093 & 43.0 & \\
100 & 6 & 0.16 & 37.5 & 4.8 \\
100 & 0 & 0.19 & 0 & \\
600 & 1 & 0.14 & 7.1 & 375.5 \\
600 & 0 & 0.14 & 0 & \\
600 & 1 & 0.14 & 7.1 & \\
1000 & 89 & 0.15 & 593.3 & \\
1000 & 10 & 0.16 & 62.5 & \\
1000 & 80 & 0.17 & 470.6 & \\
2500 & 1 & 0.12 & 8.3 & \\
2500 & 110 & 0.14 & 785.7 & \\
2500 & 115 & 0.16 & 718.8 & \\
10000 & 3 & 0.042 & 71.4 & $\mathbf{6 1}$ \\
10000 & 4 & 0.044 & 91.0 & \\
10000 & 10 & 0.051 & 196.1 & \\
& & & & \\
\end{tabular}

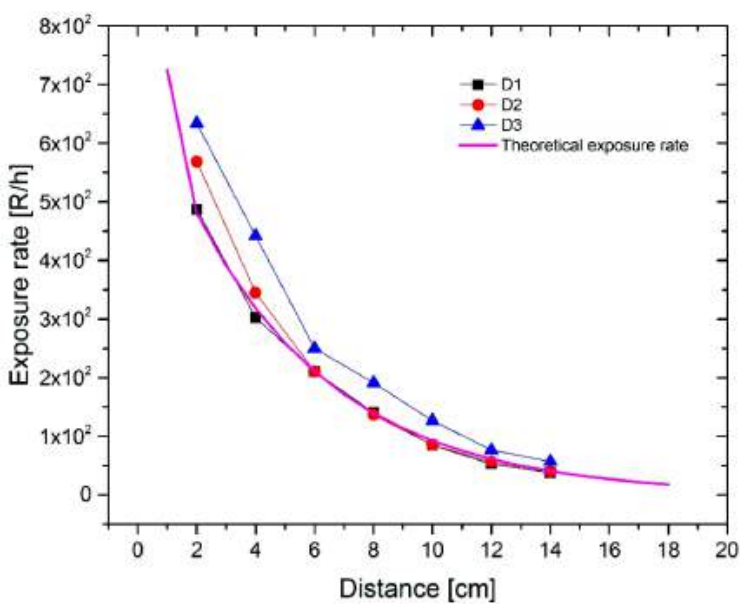

Figure 8: Exposure rate as a function of distance measured for photons using DLDs. Also shown a theoretical calculation using eq. 4 . 
Table 3: Dose equivalent at $26 \mathrm{~cm}$ from the source.

\begin{tabular}{ccccc}
\hline $\begin{array}{c}\text { Energy } \\
{[\mathrm{keV}]}\end{array}$ & Bubbles & $\begin{array}{c}\text { Sensitivity } \\
\text { bub/ } \mu \mathrm{Sv}\end{array}$ & $\begin{array}{c}\mathrm{H} \\
H[\mu \mathrm{Sv}]\end{array}$ & {$[\mu \mathrm{Sv}]$} \\
\hline 10 & 1 & 0.1 & 10 & 19.4 \\
10 & 3 & 0.11 & 27.3 & \\
10 & 4 & 0.19 & 21.1 & \\
100 & 6 & 0.093 & 64.5 & 38.2 \\
100 & 8 & 0.16 & 50.0 & \\
100 & 0 & 0.19 & 0 & 19.0 \\
600 & 1 & 0.14 & 7.1 & \\
600 & 3 & 0.14 & 21.4 & \\
600 & 4 & 0.14 & 28.6 & \\
1000 & 193 & 0.15 & 1286.7 & \\
1000 & 12 & 0.16 & 75.0 & \\
1000 & 198 & 0.17 & 1164.7 & \\
2500 & 2 & 0.12 & 16.7 & \\
2500 & 173 & 0.14 & 1235.7 & \\
2500 & 113 & 0.16 & 706.3 & \\
10000 & 1 & 0.042 & 23.8 & 54.7 \\
10000 & 1 & 0.044 & 22.7 & \\
10000 & 6 & 0.051 & 117.6 & \\
& & & $\mathbf{m S v} / \mathbf{h}$ & $\mathbf{2 . 1 6}$ \\
\hline
\end{tabular}

Measurements of Neutrons In A Mixed GammaNeutron Field Using Three Different Types of Detectors

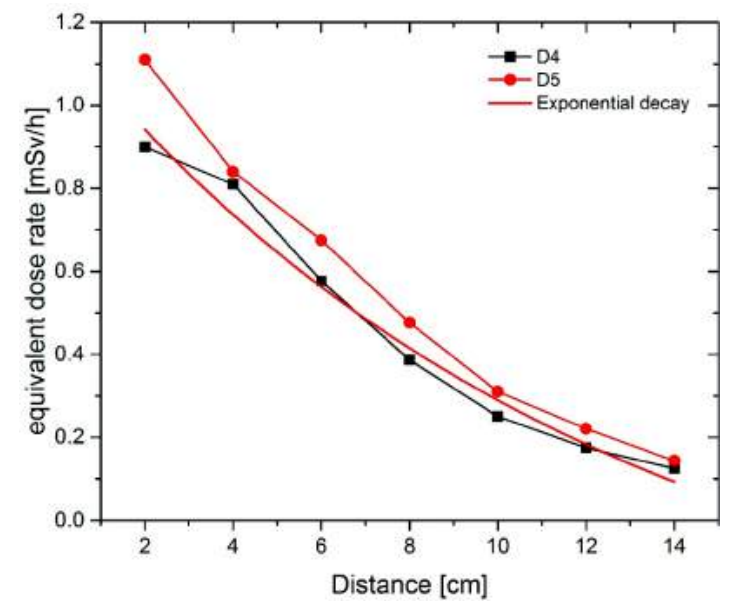

Figure 9: Exposure rate as a function of distance measured for neutrons using DLDs. 
Leal, B

Castillo, F

Gutierrez, J

Golzarri, JI

Gamboa-deBuen, I

Espinosa, G

Martínez, $\mathrm{H}$

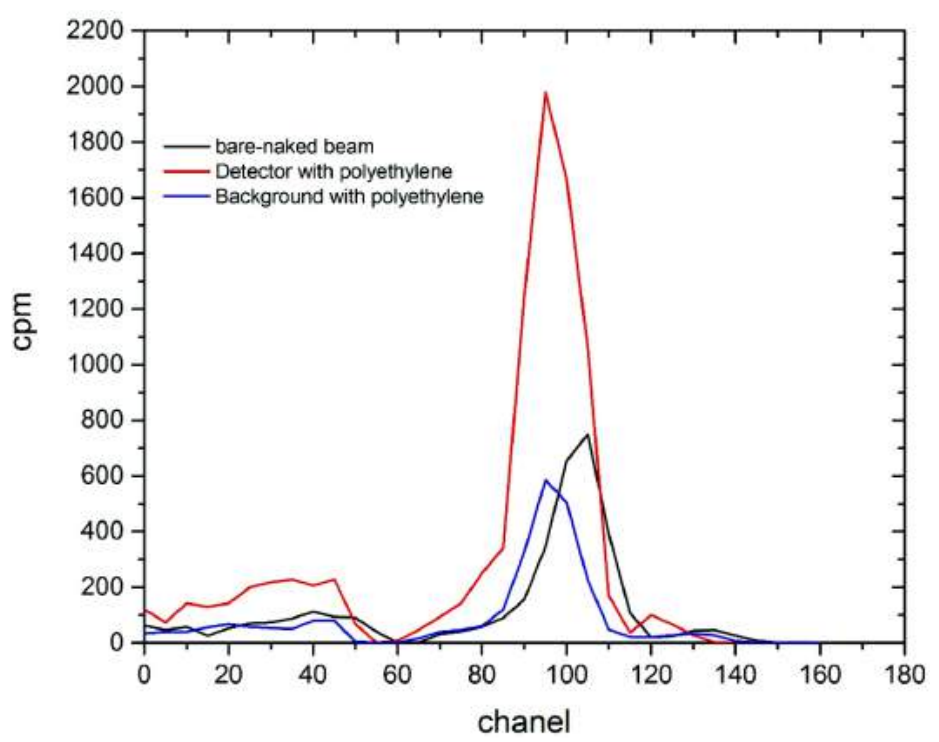

Figure 10: Spectral shape from thermal neutrons acquired with a BF3 detector.

detector readings coincide, since fast neutrons are thermalized and increase after the polyethylene shielding, for the case of the detector with polyethylene the values are higher because the cover that surrounds the detector moderates more neutrons.

\section{CONCLUSIONS}

In the case of the neutron spectrometry system, it has the disadvantage of not being calibrated with another neutron source; in addition, the background of a $5 \mathrm{Ci}$ source is intense, obscuring the signal of interest. DLDs provide fast information, but in the case of thermal neutron detectors cannot be compared with some analytical calculation. The mono channel system will be useful when the counts can be compared with some dosimetric magnitude. The system that provided most information was the bubble detectors, in addition to obtaining a neutron spectrum, the dose equivalent was determined near the source and outside its shield. It was found that it is safe to use the Am241/Be9 source if it is in the water container.

\section{ACKNOWLEDGES}

This work was partially supported by PAPIIT-DGAPA-UNAM Project IN103316. 


\section{REFERENCES}

[1] ICRP103, 2007 International Commission on Radiological Protection. The 2007 Recommendations of the International Commission on Radiological Protection. Publication 103, Annals of the ICRP 37(2-4) (Oxford: Pergamon Press) (2007)

[2] Neutron-gamma discrimination with stilbene and liquid scintillators, PerkinElmer Instruments-ORTEC, report 39580589 (1998)

[3] Dickens J. et. al., An NE213-Scintillatior-based neutron detection system for diagnostic measurements of energy spectra for neutrons having energies greater than $0.8 \mathrm{MeV}$ created during plasma operation at the Princeton Tokomak Fusion test reactor, Oak Ridge National Laboratory Central National Library ORNL/ TM-9561, (1985)

[4] Scherzinger, J, et. al., Tagging fast neutrons from an Am241/Be9 source, Applied Radiation and Isotopes 98, 74-79, (2015)

[5] Improving Neutron Dosimetry Using Bubble Detector Technology, M. A. Buckner, (2006)

[6] Bubble Technology Industries (BTI), Product Catalog, Chalk River, Ontario, Canada. October (2005)

[7] Falcão, R.C., Alexandre P. J. and DeAlmeida C.E., Neutron spectrometry with bubble detectors, International Nuclear Atlantic Conference - INAC 2009, ISBN: 978-85-99141-03-8, (2009)

[8] Ramalho E., Reina L., da Silva A.X. and Facure A., Bubble detector's evaluation for neutron field measurements in a much known source, International Nuclear Atlantic Conference - INAC 2011, ISBN: 978-85-99141-04-5, (2011)

[9] Bonin H.W., Desnoyers G.R., and Cousins T., Fast neutron dosimetry and spectroscopy using bubble detectors, Radiation Protection Dosimetry, Vol. 46, No. 4, pp. 265-271 (1993)

[10] BF 3 Neutron Detector Tests. U. S. Department of energy, Pacific Northwest, National Laboratory. December 9, (2009)

[11] Sharpe J., Salmon P.G., Characteristics of BF 3 Counters and Precautions to be adopted in their use, A.E.R.E. Report EL/R 1073 (1952)

[12] Reglamento general seguridad radiológica (Publicado en el D.O.F. del 22 de noviembre de 1988) (1989)
Measurements of Neutrons In A Mixed Gamma-

Neutron Field

Using Three

Different Types of

Detectors

\section{$\longrightarrow$}

